\title{
The Effects of Parental Behavior on Infants' Neural Processing of Emotion Expressions
}

\author{
Samantha Taylor-Colls and R. M. Pasco Fearon \\ University College London and Developmental Neuroscience Unit, Anna Freud Centre, London
}

\begin{abstract}
Infants become sensitive to emotion expressions early in the 1st year and such sensitivity is likely crucial for social development and adaptation. Social interactions with primary caregivers may play a key role in the development of this complex ability. This study aimed to investigate how variations in parenting behavior affect infants' neural responses to emotional faces. Event-related potentials (ERPs) to emotional faces were recorded from 40 healthy 7-month-old infants ( 24 males). Parental behavior was assessed and coded using the Emotional Availability Scales during free-play interaction. Sensitive parenting was associated with increased amplitudes to positive facial expressions on the face-sensitive ERP component, the negative central. Findings are discussed in relation to the interactive mechanisms influencing how infants neurally encode positive emotions.
\end{abstract}

There is a great deal of current scientific interest in the impact of early experience on brain development (Belsky \& De Haan, 2011). Studies of extreme deprivation occurring during childhood, such as maltreatment or institutional rearing, have yielded consistent evidence demonstrating a range of effects on several structural and functional brain parameters. For example, there is evidence to suggest that institutionally reared children have larger amygdala volumes compared to family-reared children (Mehta et al., 2009), with the length of stay in institutions also accounting for variation in amygdala volumes within this group (Tottenham et al., 2010). Furthermore, research has indicated that past maltreatment is associated with reduced hippocampal volume in adults, and that children exposed to maltreatment show reduced corpus callosal volume (for a review, see McCrory, De Brito, \& Viding, 2012). Furthermore, several studies have shown that maltreated children, relative to controls, display increased neural responsivity to angry facial expressions, as well as parallel biases in attention to, and categorization of, angry faces (Pollak \& Kistler, 2002; Pollak, Klorman, Thatcher, \& Cicchetti, 2001; Shackman, Shackman, \&

We would like to thank the parents and infants for participating in the study. We would also like to thank Tarik Bel-Bahar, Lara Platten, and Mikolaj Hernik for their help in running the study, as well as James Sheffield and Hannah Pincham for their invaluable input.

Correspondence concerning this article should be addressed to R. M. Pasco Fearon, Research Department of Clinical, Educational and Health Psychology, Gower Street, London WC1E 6BT, UK. Electronic mail may be sent to p.fearon@ucl.ac.uk.

The copyright line for this article was changed on Feb 19, 2015 after original online publication.
Pollak, 2007). While these studies are of exceptional importance for understanding the impact of extreme deprivation, and represent proof-of-principle that early environments can be shown to influence brain function, they do not shed light directly on whether and how the vast majority of variability in parental care occurring outside these extremes influences brain development. This is of vital importance because the quality of early parental care, particularly the extent to which that care is sensitive and responsive to the infant's cues and communications, is known to be an important influence on children's social and emotional development (e.g., NICHD Early Child Care Research Network, 1999). Measuring neural activity in infants has become increasingly feasible with the use of electroencephalography (EEG). This safe and noninvasive method of assessing the brain's spontaneous and induced neural activity by recording electrical signals at the scalp, allows for the reliable measurement of numerous neurocognitive processes. Using EEG methodology, the current report examines the relation between directly observed patterns of parent-child interaction and infants' neural responses to highly salient socioemotional cues, emotional faces.

\footnotetext{
(C) 2015 The Authors

Child Development published by Wiley Periodicals, Inc. on behalf of Society for Research in Child Development.

This is an open access article under the terms of the Creative Commons Attribution-NonCommercial License, which permits use, distribution and reproduction in any medium, provided the original work is properly cited and is not used for commercial purposes.

All rights reserved. 0009-3920/2015/8603-0015

DOI: $10.1111 /$ cdev. 12348
} 
Faces are exceptionally important signals for infants to detect and learn about in the 1st year of life, and past research has demonstrated that infants possess early-appearing and rapidly developing abilities to do so. For example, infants preferentially orient to face-like stimuli at birth (e.g., Johnson, Dziurawiec, Ellis, \& Morton, 1991; Pascalis \& Kelly, 2009) and recognize their mother's face within the first few days of life (Pascalis \& de Schonen, 1994). Clear evidence that infants distinguish emotional expressions in face stimuli can been found between 5 and 7 months of age (e.g., Peltola, Leppänen, Mäki, \& Hietanen, 2009). A considerable amount is known about the neural processes accompanying these functional capabilities for processing emotion in infants, derived largely from studies employing EEG. Numerous studies have consistently identified three key event-related EEG components in infants that are sensitive to face stimuli and that may also have emotion-sensitive properties: the N290, the P400, and the negative central (Nc) components. The most studied and consistent neural correlate of emotion processing in infancy is the Nc, a negative deflection occurring over central sites between 300 and $600 \mathrm{~ms}$ after stimulus onset. The Nc has repeatedly been found to differentiate between positive and negative (usually fearful) emotions (De Haan \& Nelson, 1998; Nelson \& De Haan, 1996) and is thought to reflect the activation of attentional processing systems linked to the appraisal of the motivational significance of emotional expressions (De Haan, 2007). In particular, the amplitude of the Nc tends to be greater in response to fearful emotional expressions than positive or neutral emotions (De Haan, Belsky, Reid, Volein, \& Johnson, 2004; Leppänen, Moulson, Vogel-Farley, \& Nelson, 2007; Peltola et al., 2009). Much less work in infancy has examined the Nc in relation to angry or sad faces, but what studies exist indicate that, for angry faces at least, a larger Nc is observed, relative to happy or neutral emotional expressions (Grossmann, Striano, \& Friederici, 2007; Hoehl \& Striano, 2008).

There are at least two lines of evidence that provide the basis for the hypothesis that neural systems subserving emotion processing in early life may be affected by variation in parental care outside of frank abuse or severe deprivation. First, there is substantial evidence that infants of depressed mothers show greater relative right frontal EEG activation in the alpha frequency range than infants of nondepressed mothers (e.g., Dawson, Klinger, Panagitodes, Hill, \& Spieker, 1992; Jones, Field, \& Almeida, 2009). This hemispheric EEG bias appears to be associated with differences in emotion, particularly negative affect and withdrawal, both in adults and children (Davidson, 1995; Fox, 1994). Although one cannot conclude with certainty that asymmetries in alpha activation are linked to neural processes specifically involved in the processing of emotional stimuli (such as facial expressions), Davidson and Fox (1982) found that infants show transient EEG shifts toward greater relative right alpha activation when processing negative emotion expressions. Furthermore, this same stimulus-linked asymmetry appears to be enhanced in infants of depressed mothers when observing negative emotional faces compared to positive emotional faces, particularly those infants whose mothers had been observed to behave intrusively (Diego et al., 2002). A recent study provides more direct evidence of differential facial emotion processing in infants related to maternal depression (Forssman et al., 2014). The results of this behavioral study indicated that maternal depressive symptoms were associated with infants' decreased attentional disengagement from fearful facial expressions relative to happy or neutral expressions, as measured using eye tracking (Forssman et al., 2014). This main effect of maternal depression was also qualified by an interaction with a genetic variant in the promoter region of the tryptophan hydroxylase gene (TPH2); those infants with the $\mathrm{T}$ allele of this gene who had also been exposed to relatively high maternal depressive symptoms showed the greatest tendency for reduced disengagement of gaze from fearful faces. Finally, a recent structural imaging study of 10 -year-olds found that maternal depressive symptoms experienced across childhood were associated with larger amygdala volumes, a brain region closely involved in emotional information processing (Lupien et al., 2011).

While we cannot rule out the possibility that other factors might be involved, particularly genetically influenced affective tendencies shared by mother and child, a disturbed pattern of parentchild interaction is a plausible explanation for these differences in emotion-related neural activity, attentional disengagement, and possibly even brain structure, in the offspring of mothers with depressive disorder or depressive symptoms. Consistent with the assertion that parental behavior may play a key role, Hane and Fox (2006) found that directly observed parenting insensitivity was associated with greater relative right frontal EEG asymmetry in 9-month-old infants. This study arguably provides the strongest current evidence of parenting effects on infant brain function.

Thus, there is convergent evidence that maternal depression may be associated with changes in young 
children's processing of emotion in offspring, and there is good indirect evidence that this may be linked to disturbances in parent-child interactions. Furthermore, postnatal depression appears to be associated with a sensitization of neural systems linked to negative emotion in particular, although whether, and indeed which, parenting processes are responsible for this sensitization remains to be thoroughly explored. Given that the features of parenting that are affected by postnatal depression-particularly hostile, intrusive, and/or withdrawn parenting - are by no means restricted to it, it is plausible to expect that variation in parenting in nondepressed samples would be associated with measurable differences in infant neural correlates of emotion processing, although to date no study has directly tested this.

A second source of evidence that parental care may influence the neural development of emotion processing comes from a study by De Haan et al. (2004) who examined the relation between maternal personality and infant event-related potentials (ERPs) to emotional faces. These authors chose maternal personality as an indirect marker for the infant's exposure to different patterns of parental care in light of the extensive evidence that personality influences parenting (Belsky \& Jaffee, 2006; Prinzie, Stams, Dekovic, Reigntjes, \& Belsky, 2009). De Haan et al. found that infants of mothers who scored high on positive affect showed greater Nc amplitudes to negative, relative to positive, emotion expressions, although only when the infant also scored high on a dimension of temperamental positive affect. Looking-time data also indicated that infants of mothers who scored high on positive affect also showed shorter looking times to positive emotional expressions. Given these findings, De Haan et al. interpreted their results to indicate that, in contrast to the sensitization effects described above for postnatal depression, maternal positive affect may lead to a diminished neural response to happy facial expressions, through repeated exposure within the caregiving environment. The findings are intriguing, although limited by their lack of direct observation of parenting.

In the current investigation we tested the association between directly observed parenting behavior and infant neural processing of emotion in a nonclinical sample of forty 7-month-old infants. In so doing, we sought to respond to Belsky and De Haan's (2011) call for research to "determine whether and how variation in parenting in the normal range affects the brain development of children not exposed to extreme adversity" (p. 423).
We predicted that ERPs related to facial emotion processing would be linked to variations in the quality of parental care. In order to minimize multiple hypothesis testing, we followed De Haan et al. (2004) and concentrated our analyses only on the Nc EEG component, in light of the fact that the best positive evidence currently available regarding the relation between emotion-related infant ERPs and the caregiving environment concerns the $\mathrm{Nc}$ (De Haan et al., 2004). Furthermore, the Nc is more consistently linked to emotion processing than the N290 or P400 (De Haan, Johnson, \& Halit, 2003). Overall, evidence indicates that the N290 and P400 are more closely linked to general face processing and feature detection than emotion processing per se (De Haan, Pascalis, \& Johnson, 2002; Halit, De Haan, \& Johnson, 2003; Scott \& Monesson, 2010; Scott \& Nelson, 2006), with comparisons being drawn to the adult face-sensitive ERP component the N170 (due to their similar topographical distributions and functional properties; see De Haan et al., 2003). Like the N290/P400, the adult N170 has shown inconsistent effects of emotion (Eimer \& Holmes, 2007; Eimer, Holmes, \& McGlone, 2003; Holmes, Winston, \& Eimer, 2005). The Nc, by contrast, appears to index attentional processes related to the salience, motivational significance, or familiarity of emotional stimuli (De Haan et al., 2003), and as such is a logical candidate for an environmentally sensitive neural component of emotion in early development. When measuring parenting, we examined maternal sensitivity and responsiveness in particular, because of their central importance to models of early child development (e.g., attachment theory) and their robust empirical association with a wide range of developmental outcomes. Because there is evidence for both sensitization and habituation/exposure effects of parenting on infant emotion processing, we did not advance strong directional hypotheses. Finally, in light of De Haan et al.'s (2004) findings we explored possible interactions between parental sensitivity and infant temperament in infants' neural responses to emotional faces.

\section{Method \\ Participants}

Mothers and their healthy 7-month-old infants were recruited from the community. Seventy-seven 7-month-old infants $\left(M_{\text {age }}=229\right.$ days; 40 males, 37 females) and their mothers participated in this study. All infants were born full term (i.e., 37- 
42 weeks) and of normal birth weight. Of the 77 infants who participated, $40 \quad\left(M_{\text {age }}=230\right.$ days, $S D=9.8,17$ females, $82 \%$ Caucasian) had usable EEG data. The remaining 37 infants were excluded due to fussiness/inattentiveness and excessive ERP artifact including eye and body movement. This attrition rate is consistent with other infant ERP studies (De Haan et al., 2004; Leppänen et al., 2007). Notably, the infants who were excluded did not significantly differ from those who were included in terms of temperament, child gender, maternal age, family household income, maternal occupation, or maternal education (lowest $p=.24$ ). Infants needed to have artifact-free EEG data on at least 10 trials per emotional expression condition to qualify for further data analysis.

\section{Procedure}

Participating mother-infant dyads visited the laboratory when their infants were 7 months old. The EEG recording sessions on average lasted 20 min including net application and a recording of $3 \mathrm{~min}$ of resting data. Infants were securely seated in an infant car seat in a soundproof room, with viewing distance $50 \mathrm{~cm}$ from a $10.5 \times 13.5 \mathrm{in}$. screen. The experiment consisted of 210 face trials (70 happy, 70 fearful, and 70 neutral). Each trial was shown for a duration of $500 \mathrm{~ms}$, presented in a random order. The interstimulus interval varied randomly between 500 and 1,000 ms. Trials began once the infant's attention was drawn to the screen. Infants' behavior was monitored via video camera. If the infant became fussy, a colorful animation with sound was activated to redirect the infant's attention. When an infant's attention could no longer be redirected to the monitor, the session was terminated. Parents were instructed to not interact with their infant during the session. After a short break, mothers and their infants were videotaped while playing together in a purpose-built babyfriendly room. Mothers were instructed to play with their infant as they usually would at home. The first $3 \mathrm{~min}$ included a free-play interaction without toys followed by a further 3-min free-play interaction with toys.

\section{Emotion Stimuli}

The stimuli consisted of five female Caucasian actors each posing a happy, neutral, or fearful face (210 total trials). We chose fear as a negative emotional stimulus because the great majority of infant emotional face-processing studies have examined this emotion (e.g., De Haan et al., 2004; Leppänen et al., 2007; Peltola et al., 2009) and as such the Nc response has been better characterized in infant populations than other negative emotions, such as anger or sadness. The faces were shown against a white background and were cropped so no hair or other features could be seen. The static images were centrally positioned and measured $12 \times 9 \mathrm{~cm}$ on a $10.5 \times 13.5 \mathrm{in}$. screen. The stimuli were provided by the MacBrain Face Stimulus Set. Development of the MacBrain Face Stimulus Set was overseen by Nim Tottenham and supported by the John D. and Catherine T. MacArthur Foundation Research Network on Early Experience and Brain Development.

\section{EEG Recording}

EEG was recorded continuously during the face viewing trials using a 128 hydrocel geodesic net (Electrical Geodesic Inc., Eugene, OR) referenced to the vertex $(\mathrm{Cz})$. The electrical signal was amplified with a $0.1-$ to $100-\mathrm{Hz}$ band-pass with a sampling rate of $250 \mathrm{~Hz}$. All EEG data were analyzed offline using the EEG platform EEGLAB (Delorme \& Makeig, 2004) and in-house custom-made MATLAB scripts.

\section{Data Reduction}

For each participant the EEG signal was segmented into epochs beginning $100 \mathrm{~ms}$ before stimulus onset and ending $750 \mathrm{~ms}$ after. Prior to EEG processing, the videos were examined by eye, and segments were rejected if the infant was not looking at the screen. To identify and remove bad channels and artifacts, we used the automated artifact detection system FASTER (Nolan, Whelan, \& Reilly, 2010). FASTER uses a series of processing steps that have been shown to accurately identify a broad range of EEG artifact (Nolan et al., 2010): First, bad channels are detected by rejecting channels whose mean correlation with neighboring channels is less than $-3 S D$ from the overall recording mean. Next, bad channels are identified if they have extreme $(>3 S D)$ values for their variance and Hurst exponents (a measure of long-range dependency in a signal). Following bad channel identification, epochs are checked for large artifacts present across channels (typically due to movements) by removing epochs in which the amplitude range, average amplitude, or average channel variance across an epoch was $>3 S D$ from the average across all epochs. Next, independent components analysis (ICA) is performed using the infomax algorithm. 
Eye blinks and eye movements are identified by computing correlations between ICs and eye channels (and rejected when correlations > $3 S D$ from the mean). Other artifact ICs are identified as those having outlying ( $>3 S D$ ) kurtosis (usually "pop-off" electrode artifact), those having a very low gradient power spectrum (identifies white noise), those having extreme Hurst exponents, or ICs with extreme temporal slopes. All artifactual components are removed and then the raw EEG data are reconstituted from the ICA weight matrix. Individual channels are screened within epochs to check for transient artifacts when a channel had extreme variance, slope, range, or average amplitude. Finally, bad channels are interpolated using spherical spline interpolation (no more than $10 \mathrm{bad}$ channels were interpolated in a segment). Once the automated process was complete, each data set was checked by eye to ensure that no clear-cut artifacts were missed by the automated algorithm. In only one case was it necessary to remove a single epoch due to missed movement artifact. Finally, the data were averaged and re-referenced to the average reference. The average number of good trials was $21(S D=8.2$, range $=10-48)$ for fearful faces, 22 $(S D=8.7$, range $=10-45)$ for happy faces, and $20(S D=7.1$, range $=10-43)$ for neutral faces. The differences in trial counts between categories were not statistically significant, $F(2,38)=2.13, p=.13$.

\section{Measures}

\section{Parenting}

The parent-infant interactions were coded using the Emotional Availability Scales (EA; Biringen, 2000; Biringen, Robinson, \& Emde, 2000). The EA is a measure that draws heavily on attachment theory, with the emphasis being on the contribution of both the parent and infant on the evolving interaction. The EA scales comprise four parental dimensions: (a) sensitivity, measured on a 9-point scale; (b) structuring, on a 5-point scale; (c) nonintrusiveness, on 5-point scale; and (d) nonhostility, on a 5-point scale. The EA scales have been shown to robustly predict infant attachment classifications (Easterbrooks, Biesecker, \& Lyons-Ruth, 2000; Ziv, Aviezer, Gini, Sagi, \& Koren-Karie, 2000) and have been demonstrated to be stable from 6 to 12 and 20 months of age (Ziv, Gini, Guttman, \& Sagi, 1997). The four scales were quite strongly intercorrelated $(r s=0.89-0.50)$. In order to reduce the number of variables for analysis and create a more reliable overall index of sensitivity, the four scales were standardized and summed (Cronbach's $\alpha=.81$ ). The interactions were coded by a single coder who was blind to the EEG data. Independent reliability coding of $25 \%$ of the data showed acceptable reliability (individual scale interrater reliability coefficients were intraclass correlation coefficients $[$ ICCs $]=0.71-0.75$, and the overall composite scale interrater reliability was 0.80 ).

\section{Infant Temperament}

Due to the possible role that temperament may play in the processing of emotional faces, we collected data on infant temperament using the Infant Behaviour Questionnaire (IBQ; see Rothbart, 1981). The IBQ assesses the frequency of specific temperamental behaviors that have been observed in the last week and results in six temperamental dimensions: activity level, smiling and laughing, distress, and latency to approach sudden or novel stimuli distress to limitations, soothability and duration of orienting. In this study, we were interested in indices related to negative and positive affect, and hence we selected the following scales: distress and latency to approach, distress to limitations, soothability, and smiling and laughter. The two distress scales correlated quite highly $(r=.51)$ and were therefore standardized and summed to create a "distress" index. High internal consistency across all six dimensions has been demonstrated at 3, 6, 9, and 12 months of age (Rothbart, 1981).

\section{Selection of Nc EEG Component}

Consideration of past studies' component timings guided the selection of the time window for the Nc. The Nc was defined as the average of the negative EEG deflection occurring between 350 and $650 \mathrm{~ms}$ poststimulus onset. The Nc is reported to be most prominent over central sites (De Haan et al., 2004; Leppänen et al., 2007) and the following electrode clusters were selected for each hemisphere; left: 35, 41, 36, 47, 42; right: 110, 104, 103, 93, 98 (see Figure 1).

\section{Data Analysis}

The analysis of Nc amplitude involved the use of mixed 2 (hemisphere, left vs. right) $\times 3$ (emotion, fear, happy, neutral) analyses of variance, with sensitivity or temperament entered as continuous covariates. Correlational and regression analyses were used to follow up any sensitivity or temperament effects, based on a subtraction of average Nc ampli- 


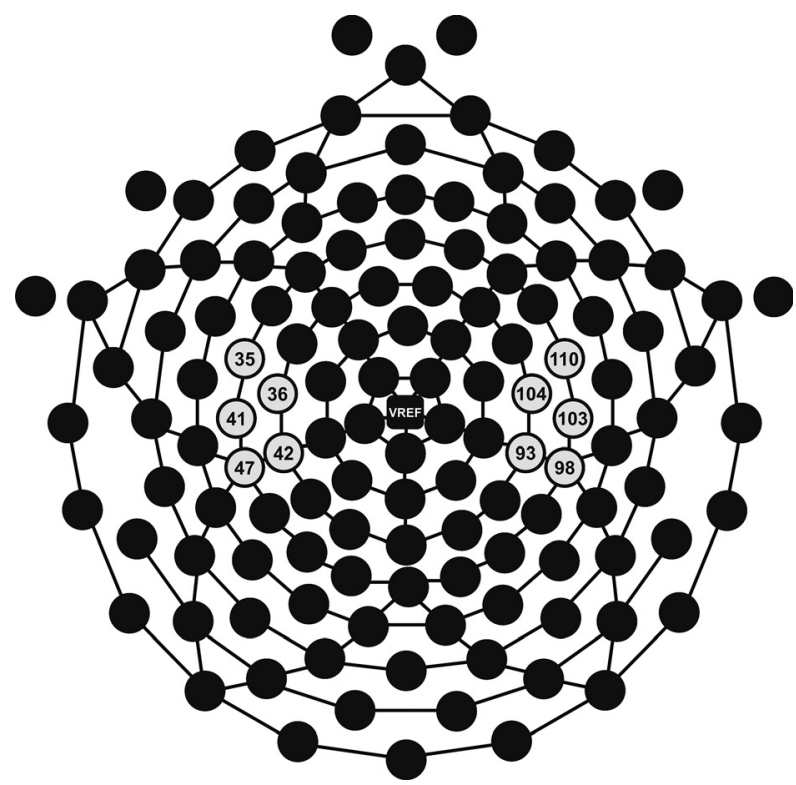

Figure 1. Electrode clusters selected for the negative central. The two electrode clusters used for analysis are highlighted in white.

tudes for emotional faces from neutral faces (fearneutral; happy-neutral). We chose this contrast because our main hypotheses focused on neural responding to emotion, and hence the neutral/nonemotional face stimuli represented the most pertinent point of reference. In the results, we show that using an alternative coding (fear-mean [happy-neutral]; happy-mean [fear-neutral]) does not materially affect the results. Interactions between temperament and sensitivity were tested using regression, in which interactions were computed as the product of the linear effects of each variable (Temperament $\times$ Sensitivity).

\section{Results}

The results are presented in three sections. In the first, the effect of emotion (happy, fear, neutral), laterality, and maternal sensitivity are examined. In the second, we present analyses of temperament. In the final section, we test for Sensitivity $\times$ Temperament interactions.

\section{Effects of Emotion, Laterality, and Sensitivity on Nc Amplitude}

The main effect of emotion was statistically significant, indicating that mean Nc amplitudes differed according to the emotion of the face stimuli, $F(2,76)=3.55, p=.034, \eta_{\mathrm{p}}^{2}=.085$. Consistent with previous findings, fearful faces elicited larger amplitudes $(M=-4.78)$ than happy $(M=-3.73)$ and neutral $(M=-3.50)$ faces (see Figure 2$)$. The main effects of laterality, $F(1,38)=1.30, p=.26, \eta_{p}^{2}=$ .033 , and sensitivity, $F(1,38)=0.55, p=.462$, $\eta_{\mathrm{p}}^{2}=.014$, were not significant. However, there was a significant Emotion $\times$ Sensitivity interaction, $F(2,76)=3.39, p=.039, \eta_{p}^{2}=.082$. To tease apart this interaction, we created two difference scores reflecting the amplitude of each emotional face relative to neutral faces (fear-neutral, happy-neutral) and correlated each of these with sensitivity. The relative Nc amplitude for fearful faces did not correlate with sensitivity $(r=-.071, p=.67)$, but the relative Nc amplitude for happy faces did $(r=-.41, p=.008)$. In other words, higher maternal sensitivity was associated with a larger, more negative, Nc for happy faces, relative to neutral faces. Notably, this correlation was maintained after controlling for infant gender $(r=-.40, p=.009)$.

A scatterplot showing this association is presented in Figure 3. Two participants' ERP difference scores (either fear-neutral or happy-neutral) were outliers, and these difference scores exceeded $2.5 S D$ from the group mean. We therefore repeated the above analyses, excluding the two outliers. Importantly, the statistical significance of the results remained the same (and effect sizes increased) once these participants were removed from the analysis: emotion main effect, $F(2,72)=6.04, p=.004$, $\eta_{\mathrm{p}}^{2}=.114 ;$ Emotion $\times$ Sensitivity interaction $F(2$ $72)=4.01, p=.022, \eta_{\mathrm{p}}^{2}=.100$; correlation between

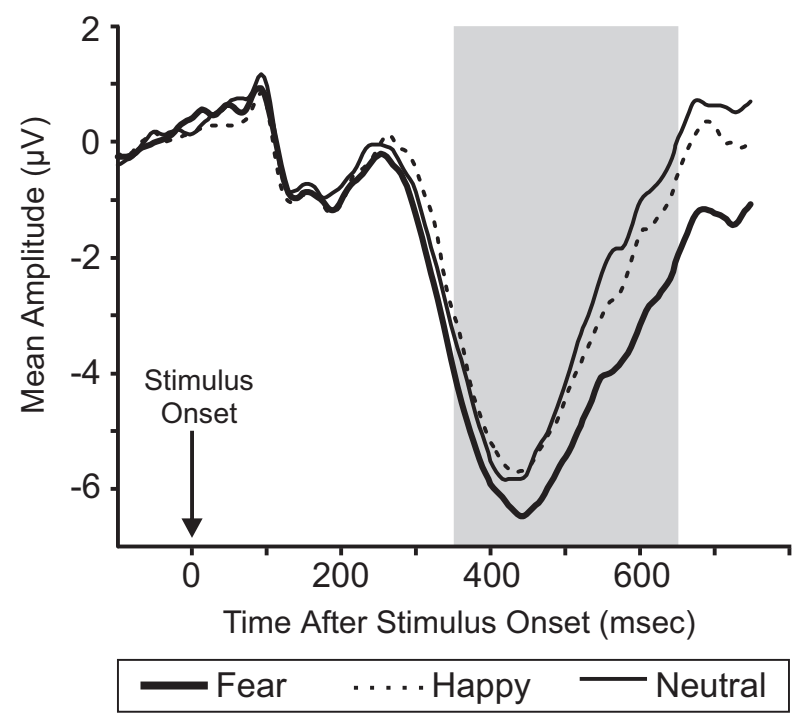

Figure 2. Event-related potential wave form for negative central (Nc) electrode cluster. Nc time window shaded in gray. 


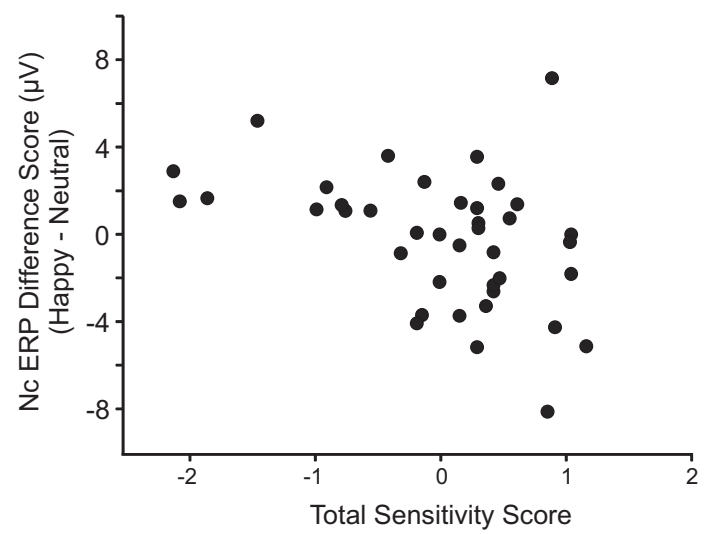

Figure 3. Scatterplot of happy minus neutral Nc average amplitude as a function of maternal sensitivity. $\mathrm{Nc}_{\mathrm{c}}=$ negative central component; $\mathrm{ERP}=$ event-related potential; $y$-axis measures differences in average amplitude between happy and neutral faces in microvaults.

sensitivity and happy-neutral ERP difference score, $r=-.52, p=.001$. Furthermore, the correlation was also unchanged when the number of trials in each emotion category was partialed out (with outliers: partial $r=.41, p=.011$; without outliers: partial $r=-.48, p=.004)$. Finally, the correlation was also unchanged when the difference scores were recomputed as a contrast between the Nc for happy faces relative to the average Nc for fearful and neutral faces (with outliers: $r=.44, p=.005$; without outliers: $r=.45, p=.005)$.

\section{Temperament Effects}

When we examined the average Nc amplitude in relation to temperamental negative affect/distress, no significant main effects or interactions were found. However, significant effects were found for infant soothability. Specifically, the Soothability $\times$ Emotion interaction was significant, $F(2,74)=4.58, p=.014$. When we examined the correlations between soothability and the Nc ERP difference scores, we found a significant correlation for the relative Nc amplitude for fearful faces $(r=.38, p=.017)$ but not happy faces $(r=.01$, $p=.97)$. The direction of the correlation implies that infants with lower soothability ratings had a larger (more negative) Nc amplitude to fearful faces, relative to neutral faces. Notably, this association was maintained after controlling for gender $(r=.40$, $p=.014)$, when the two outlying scores were removed $(r=.41, p=.010)$ and when trial count was partialed out (with outliers: partial $r=.37$, $p=.023$; without outliers: partial $r=.39, p=.020$ ).
Furthermore, recomputing the difference score so that the amplitude to fearful faces was measured relative to both happy and neutral faces (i.e., fear minus the average of happy and neutral faces), did not appreciably alter the findings $(r=.44, p=.005)$.

There was also a significant Soothability $\times$ Laterality interaction, $F(1,37)=5.42, p=.025$. Lower soothability ratings were associated with a larger, more negative, Nc in the left hemisphere than the right. No significant effects were found for infant positive affect (smiling and laughter).

\section{Temperament $\times$ Parental Sensitivity Interactions}

In order to test for interactions between temperament and parental sensitivity we entered main effects and product terms (Temperament $\times$ Sensitivity) as independent variables into regression analyses, with the relative Nc to fearful and happy faces (vs. neutral) as two separate dependent variables. These analyses were conducted separately for infant negative affect, soothability, and positive affect. No significant interactions were found.

\section{Discussion}

There has been increasing interest in the role of the environment in shaping neural development (Belsky \& De Haan, 2011). Animal studies and studies of children exposed to extreme circumstances, such as institutional care or maltreatment, have provided quite good initial evidence regarding the functional (and in some cases structural) brain changes linked to highly adverse environments. Much less has been done to identify how more normative variations in the quality of early experience might influence brain development. In light of the highly consistent evidence concerning the developmental impact of variations in the sensitivity of parental care on emotional and behavioral development (e.g., NICHD Early Child Care Research Network, 1999), understanding the neural systems influenced by this particular aspect of the early environment is an important goal for developmental research.

As a first step toward that goal, we investigated 7-month-old infants' neural responses to emotional expressions. Emotional faces are a very useful way to study infant brain responses in this context because a great deal of research has already characterized the typical ERPs evoked by faces and by emotional expressions in particular. Furthermore, and equally importantly, emotional facial expres- 
sions are of great significance as cues and social signals embedded within parent-infant interactions (Walden \& Ogan, 1988) that infants are sensitive to from early in life (Field \& Walden, 1982). Furthermore, there is good evidence at later stages of development that emotional face processing is altered among children and adults with behavioral and affective disorders (e.g., Blair, Colledge, Murray, \& Mitchell, 2001; Dolan \& Fullam, 2006; Sinzig, Morsch, \& Lehmkuhl, 2008).

As expected, we recorded larger Nc amplitudes in response to fearful faces compared to happy and neutral faces. Our findings add to an extensive body of literature demonstrating the sensitivity of the Nc to differences in facial emotion expression. Most importantly, when we examined the magnitude of the $\mathrm{Nc}$ for each emotional expression in relation to objectively assessed parental sensitivity, we found a marked association for happy, but not fearful, facial expressions. Infants of mothers who were more sensitive and responsive during their interactions showed larger Nc neural responses to happy faces, relative to neutral faces. The magnitude of the association was relatively large and sensitivity analyses suggested that the result was not driven by a small number of data points at the tails of the bivariate distribution, or by procedural factors like variability in trial counts or how the emotion contrasts were computed in the analysis. The finding would seem to suggest that sensitive parental responses to the infant's cues during interactions lead the developing brain to encode or evaluate emotional expressions differently, perhaps attaching greater motivational value to them. The finding is intriguing given the crucial role of positive emotional expressions in eliciting and reinforcing approach behavior, social engagement, and social reward (e.g., Lin, Adolphs, \& Rangel, 2012; Sorce, Emde, Campos, \& Klinnert, 1985). We could speculate that during the course of warm, responsive, and contingent (and therefore predictable) interactions, the infant comes to encode positive emotional expressions as stronger cues to rewarding social interaction than those that experience less contingent, reciprocal, and warm interactions. Another possibility of course is that sensitive parenting interactions may simply involve greater exposure, on the part of the infant, to positive emotional expressions, which then alters the infant's neural responding to that stimulus. One might question this interpretation because the effect we observed would seem more consistent with sensitization than habituation, in that higher sensitivity was associated with larger, not smaller, Nc amplitudes. However, there is evidence that repeated exposure can, under certain circumstances, heighten rather than diminish infant attention to a range of stimuli (e.g., Roder, Bushnell, \& Sasseville, 2000). Furthermore, despite the fact that the Nc is larger for fearful than happy or neutral faces (which should be more familiar), the $\mathrm{Nc}$ is also larger to mother's face than a stranger's, and to familiar relative to unfamiliar objects (De Haan \& Nelson, 1997, 1999; Nelson et al., 2000). Direct measurement of the frequency with which infants are exposed to different parental facial emotion expressions would be invaluable in disentangling these two possibilities.

The overall pattern of our findings was quite different from those of De Haan et al. (2004). Not only did they not find any main effect of their indirect "marker" of parenting (parental personality) on the Nc for happy or fearful faces, where they did find an association it was in relation to Nc amplitudes to fearful, not happy, faces. In their study, among highly positive infants, higher maternal selfreported positive affect was associated with larger infant Nc amplitudes to fearful faces, which was interpreted as evidence that this expression may have been more novel for these infants. It is also notable that their study found interactions between temperament and maternal personality, while our study found no parallel evidence of such interactions in relation to maternal sensitivity. The two studies differ in numerous ways, however, so direct comparisons are not possible. The key difference of course is that the measures of the environment were very different: De Haan et al. relied on a measure of personality, while the current study measured distinct parenting behaviors directly. In addition to that crucial difference, De Haan et al. also did not analyze differences in EEG amplitude to neutral face stimuli, which may have limited the power of the statistical analyses to detect the relevant effects. Notably, both the De Haan et al. study and ours found evidence that temperamental dimensions broadly related to negative affect were associated with heightened Nc amplitudes to fearful faces. However, although soothability - the temperamental dimension linked to Nc amplitude in this study - is linked to negative emotion, it is by no means a direct assessment of negative emotionality, and as such our finding is clearly not a precise replication. Finally, it was notable that we did not find Temperament $\times$ Parenting interactions in this investigation, although the low power of tests of statistical interaction may be an important consideration in that regard. 
It is important to consider why it might be that we did not find any association between parental sensitivity and infant neural responding to fearful faces. We did not advance a specific hypothesis about the role of different emotions and the specificity of the effect for happy faces was not anticipated. Several explanations are worth considering. First, positive emotional expressions are much more prevalent during early interactions than fearful ones (Malatesta \& Haviland, 1982), and this, in and of itself, is a highly plausible explanation for the differential findings. If the effect we observed reflects a process of learning taking place during caregiving interactions, then the results may not be surprising, particularly in a low-risk sample, because positive expressions are likely to be the subject of more learning in the first half of the 1st year of life than fearful expressions. As noted already, it was notable that when we examined temperament we did find quite robust associations with $\mathrm{Nc}$ amplitudes to fearful faces, with infants scoring lower on soothability demonstrating larger neural responses to fear. Thus, it was not obviously the case that fearful faces were in some way less effective experimental stimuli (the larger neural response to fear faces suggests that the Nc is not noisier in this condition). It is tempting to speculate that this latter temperamental effect might involve a different set of mechanisms, less related to learning and less immediately susceptible to environmental input, than those underpinning the association between maternal sensitivity and infant neural responses to positive emotions. More research is clearly needed to deepen our understanding of these early developmental processes.

This is not the only study to highlight the potential importance of positive emotion in socioemotional development and in the domain of parent-child relationships in particular (e.g., Forbes \& Dahl, 2005; Kochanska \& Aksan, 1995). Intriguingly, in a recent very long-term prospective study, Moutsiana et al. (2014) found that adults who 20 years earlier had been classified as insecurely attached as infants showed altered neural responding, relative to previously secure adults, in several brain areas (dorsalmedial prefrontal cortex, rostral anterior cingulate cortex) during an emotion-regulation task specifically when upregulating positive emotion. The findings appeared to suggest that previously insecure adults had greater difficulty, and deployed greater cognitive effort, when upregulating positive emotions. Similarly, White et al. (2012) found reduced amplitude of reward-related ERPs among adolescents with insecure attachment styles. These studies collectively sug- gest that the sensitivity of parental behavior may play a particularly important role in modulating the functioning of neural systems involved in approach, social engagement, and reward. This evidence, considered alongside the findings presented in this investigation, could suggest that sensitive parenting and secure attachment significantly influence positive emotional processing, either by altering the reward value of positive emotional expressions, enhancing the regulation of positive emotion, or biasing attention to positive social signals.

The findings reported here are in need of replication. It is also important to note that this study has several limitations. First, the observational procedure used in this study, though employing a commonly used protocol and utilizing a well-validated coding scheme, focuses on one particular set of parenting behaviors within a specific context (free play), and was brief. It is therefore inevitable that it does not capture all the developmentally important variance in parenting sensitivity or in other domains of parental behavior. Furthermore, our coding was quite broadly focused, and different aspects of interactions were not coded independently. It will be important in future studies to investigate the specific interactive processes that are involved in the neural effects reported here in greater depth. Such studies would benefit from using more fine-grained coding, a broader range of observational contexts, and separate, independently coded measurements of different potentially relevant parenting dimensions. Furthermore, the sample was restricted to a relatively advantaged, middle-class population and the findings may not generalize to other populations. Further research on the neural effects of variations in parenting in more at-risk samples would be extremely valuable in the future. Also, our study reports on associations that were observed concurrently and at a specific point in development, and hence it will be important in future work to determine how stable and enduring such associations may be, and when they first arise. Finally, it is critical to note that although we have tended to interpret these findings as evidence indicative of an influence of the environment on brain function, these data are purely correlational and firm causal inferences cannot be made. In that regard, intervention studies that experimentally enhance the sensitivity of parenting would be exceptionally valuable in testing the causal nature of the associations reported here.

In summary, the current study found evidence in support of the view that the quality of early mother-infant interactions may influence infants' 
brain responses to emotional expressions, and highlights the potentially important role of infants' neural coding of positive emotional expressions in particular. Replication of the current findings would be highly valuable, and future research may fruitfully explore other features of parenting, other domains of infant social-affective brain development, and other populations. Studies will ultimately need to be done to test the causal nature of observed associations between parental care and infant brain development, in longitudinal and experimental studies.

\section{References}

Belsky, J., \& De Haan, M. (2011). Annual research review: Parenting and children's brain development: The end of the beginning. Journal of Child Psychology and Psychiatry, 52, 409-428. doi:10.1111/j.1469-7610.2010.02281.x

Belsky, J., \& Jaffee, S. (2006). The multiple determinants of parenting. In D. Cicchetti \& D. Cohen (Eds.), Developmental psychopathology: Risk, disorder and adaptation (Vol. 3, 2nd ed., pp. 38-85). New York, NY: Wiley.

Biringen, Z. (2000). Emotional availability: Conceptualization and research findings. American Journal of Orthopsychiatry, 70, 104-114. doi:10.1037/h0087711

Biringen, Z., Robinson, J. L., \& Emde, R. N. (2000). Emotional Availability Scales, 3rd edition. Attachment and Human Development, 2, 257-270.

Blair, R. J. R., Colledge, E., Murray, L., \& Mitchell, D. (2001). A selective impairment in the processing of sad and fearful expressions in children with psychopathic tendencies. Journal of Abnormal Child Psychology, 29, 491-498. doi:10.1023/A:1012225108281

Davidson, R. J. (1995). Cerebral asymmetry, emotion, and affective style. In R. J. Davidson (Ed.), Brain asymmetry (pp. 361-387). Cambridge, MA: MIT Press.

Davidson, R. J., \& Fox, N. A. (1982). Asymmetrical brain activity discriminates between positive and negative affective stimuli in human infants. Science, 218, 12351237. doi:10.1126/science.7146906

Dawson, G., Klinger, L. G., Panagitodes, H., Hill, D., \& Spieker, S. (1992). Frontal lobe activity and affective behavior of infants and mothers with depressive symptoms. Child Development, 63, 725-737. doi:10.2307/ 1131357

De Haan, M. (2007). Visual attention and recognition memory in infancy. In M. De Haan (Ed.), Infant EEG and event-related potentials: Studies in developmental psychology (pp. 101-122). New York, NY: Psychology Press.

De Haan, M., Belsky, J., Reid, V., Volein, A., \& Johnson, M. H. (2004). Maternal personality and infants' neural and visual responsivity to facial expressions of emotion. Journal of Child Psychology and Psychiatry, 45, 12091218. doi:10.1111/j.1469-7610.2004.00320.x
De Haan, M., Johnson, M. H., \& Halit, H. (2003). Development of face-sensitive event-related potentials during infancy: A review. International Journal of Psychophysiology, 51, 45-58. doi:10.1016/S0167-8760(03) 00152-1

De Haan, M., \& Nelson, C. A. (1997). Recognition of the mother's face by six-month-old infants: A neurobehavioral study. Child Development, 68, 187-210. doi:10.1111/j.1467-8624.1997.tb01935.x

De Haan, M., \& Nelson, C. A. (1998). Discrimination and categorization of facial expression of emotion during infancy. In A. Slater (Ed.), Perceptual development (pp. 287-309). Hove, UK: Psychology Press.

De Haan, M., \& Nelson, C. A. (1999). Brain activity differentiates face and object processing in 6-month-old infants. Developmental Psychology, 35, 1113-1121. doi:10.1037/0012-1649.35.4.1113

De Haan, M., Pascalis, O., \& Johnson, M. (2002). Specialization of neural mechanisms underlying face recognition in human infants. Journal of Cognitive Neuroscience, 14, 199-209. doi:10.1162/089892902317236849

Delorme, A., \& Makeig, S. (2004). EEGLAB: An open source toolbox for analysis of single-trial EEG dynamics including independent component analysis. Journal of Neuroscience Methods, 134, 9-21. doi:10.1016/j.jneumeth.2003.10.009

Diego, M. A., Field, T., Hart, S., Hernandez-Reif, M., Jones, N., Cullen, C., . . . Kuhn, C. (2002). Facial expressions and EEG in infants of intrusive and withdrawn mothers with depressive symptoms. Depression and Anxiety, 15, 10-17. doi:10.1002/da.1079

Dolan, M., \& Fullam, R. (2006). Face affect recognition deficits in personality-disordered offenders: Association with psychopathy. Psychological Medicine, 36, 15631570. doi:10.1017/S0033291706008634

Easterbrooks, M. A., Biesecker, G., \& Lyons-Ruth, K. (2000). Infancy predictors of emotional availability in middle childhood: The roles of attachment security and maternal depressive symptomatology. Attachment and Human Development, 2, 170-187. doi:10.1080/ 14616730050085545

Eimer, M., \& Holmes, A. (2007). Event-related brain potential correlates of emotional face processing. Neuropsychologia, 45, 15-31. doi:10.1016/j.neuropsychologia.2006.04.022

Eimer, M., Holmes, A., \& McGlone, F. P. (2003). The role of spatial attention in the processing of facial expression: An ERP study of rapid brain responses to six basic emotions. Cognitive, Affective, E Behavioral Neuroscience, 3, 97-110. doi:10.3758/CABN.3.2.97

Field, T., \& Walden, T. A. (1982). Production and perception of facial expressions in infancy and early childhood. Advances in Child Development and Behavior, 16, 169-211. doi:10.1016/S0065-2407(08)60070-1

Forbes, E. E., \& Dahl, R. E. (2005). Neural systems of positive affect: Relevance to understanding child and adolescent depression? Development and Psychopathology, 17, 827-850. doi:10.1017/S095457940505039X 
Forssman, L., Peltola, M. J., Yrttiaho, S., Puura, K., Mononen, N., Lehtimäki, T., \& Leppänen, J. M. (2014). Regulatory variant of the TPH2 gene and early life stress are associated with heightened attention to social signals of fear in infants. Journal of Child Psychology and Psychiatry, 55, 793-801. doi:10.1111/jcpp.12181

Fox, N. A. (1994). Dynamic cerebral processes underlying emotion regulation. Monographs of the Society for Research in Child Development, 59(2/3), 152-166. doi:10.2307/1166143

Grossmann, T., Striano, T., \& Friederici, A. (2007). Developmental changes in infants' processing of happy and angry facial expressions: A neurobehavioral study. Brain and Cognition, 64, 30-41. doi:10.1016/ j.bandc.2006.10.002

Halit, H., De Haan, M., \& Johnson, M. (2003). Cortical specialisation for face processing: Face-sensitive eventrelated potential components in 3-and 12-month-old infants. Neurolmage, 19, 1180-1193. doi:10.1016/S10538119(03)00076-4

Hane, A. A., \& Fox, N. A. (2006). Ordinary variations in maternal caregiving influence human infants' stress reactivity. Psychological Science, 17, 550-555. doi:10.1111/j.1467-9280.2006.01742.x

Hoehl, S., \& Striano, T. (2008). Neural processing of eye gaze and threat-related emotional facial expressions in infancy. Child Development, 79, 1752-1760. doi:10.1111/ j.1467-8624.2008.01223.x

Holmes, A., Winston, J. S., \& Eimer, M. (2005). The role of spatial frequency information for ERP components sensitive to faces and emotional facial expression. Cognitive Brain Research, 25, 508-520. doi:10.1016/j.cogbrainres.2005.08.003

Johnson, M. H., Dziurawiec, S., Ellis, H. D., \& Morton, J. (1991). Newborns' preferential tracking of face-like stimuli and its subsequent decline. Cognition, 40, 1-19. doi:10.1016/0010-0277(91)90045-6

Jones, N. A., Field, T., \& Almeida, A. (2009). Right frontal EEG asymmetry and behavioral inhibition in infants of depressed mothers. Infant Behavior and Development, 32, 298-304. doi:10.1016/j.infbeh.2009.04.004

Kochanska, G., \& Aksan, N. (1995). Mother-child mutually positive affect, the quality of child compliance to requests and prohibitions, and maternal control as correlates of early internalization. Child Development, 66, 236-254. doi:10.2307/1131203

Leppänen, J. M., Moulson, M. C., Vogel-Farley, V. K., \& Nelson, C. A. (2007). An ERP study of emotional face processing in the adult and infant brain. Child Development, 78, 232-245. doi:10.1111/j.1467-8624.2007.00994.x

Lin, A., Adolphs, R., \& Rangel, A. (2012). Social and monetary reward learning engage overlapping neural substrates. Social Cognitive and Affective Neuroscience, 7, 274-281. doi:10.1093/scan/nsr006

Lupien, S. J., Parent, S., Evans, A. C., Tremblay, R. E., Zelazo, P. D., Corbo, V., . . S Ségiun, J. R. (2011). Larger amygdala but no change in hippocampal volume in 10year-old children exposed to maternal depressive symptomatology since birth. Proceedings of the National Academy of Sciences of the United States of America, 108, 14324-14329. doi:10.1073/pnas.1105371108

Malatesta, C. Z., \& Haviland, J. M. (1982). Learning display rules: The socialization of emotion expression in infancy. Child Development, 53, 991-1003. doi:10.2307/1129139

McCrory, E., De Brito, S. A., \& Viding, E. (2012). The link between child abuse and psychopathology: A review of neurobiological and genetic research. Journal of the Royal Society of Medicine, 105, 151-156. doi:10.1258/ jrsm.2011.110222

Mehta, M. A., Golembo, N. I., Nosarti, C., Colvert, E., Mota, A., Williams, S. C., . . Sonuga-Barke, E. J. (2009). Amygdala, hippocampal and corpus callosum size following severe early institutional deprivation: The English and Romanian Adoptees Study Pilot. Journal of Child Psychology and Psychiatry, 50, 943-951. doi:10.1111/j.1469-7610.2009.02084.x

Moutsiana, C., Fearon, P., Murray, L., Cooper, P., Goodyer, I., Johnstone, T., . . . Halligan, S. (2014). Making an effort to feel positive: Isecure attachment in infancy predicts the neural underpinnings of emotion regulation in adulthood. Journal of Child Psychology and Psychiatry, 55, 999-1008. doi:10.1111/jcpp.12198

Nelson, C. A., \& De Haan, M. (1996). Neural correlates of infants' visual responsiveness to facial expressions of emotion. Developmental Psychobiology, 29, 577-595. doi:10.1002/(SICI)1098-2302(199611)29:7 < 577::AIDDEV3 > 3.0.CO;2-R

Nelson, C. A., Wewerka, S., Thomas, K. M., deRegnier, R.-A., Tribbey-Walbridge, S., \& Georgieff, M. (2000). Neurocognitive sequelae of infants of diabetic mothers. Behavioral Neuroscience, 114, 950-956. doi:10.1037/07357044.114.5.950

NICHD Early Child Care Research Network. (1999). Chronicity of maternal depressive symptoms, maternal sensitivity, and child functioning at 36 months. Developmental Psychology, 35, 1297-1310. doi:10.1037/00121649.35.5.1297

Nolan, H., Whelan, R., \& Reilly, R. (2010). FASTER: Fully automated statistical thresholding for EEG artifact rejection. Journal of Neuroscience Methods, 192, 152-162. doi:10.1016/j.jneumeth.2010.07.015

Pascalis, O., \& de Schonen, S. (1994). Recognition memory in 3- to 4-day-old human neonates. Neuroreport, 5, 1721-1724. doi:10.1097/00001756-199409080-00008

Pascalis, O., \& Kelly, D. J. (2009). The origins of face processing in humans: Phylogeny and ontogeny. Perspectives on Psychological Science, 4, 200-209. doi:10.1111/ j.1745-6924.2009.01119.x

Peltola, M. J., Leppänen, J. M., Mäki, S., \& Hietanen, J. K. (2009). Emergence of enhanced attention to fearful faces between 5 and 7 months of age. Social Cognitive and Affective Neuroscience, 4, 134-142. doi:10.1093/scan/ nsn046

Pollak, S. D., \& Kistler, D. J. (2002). Early experience is associated with the development of categorical repre- 
sentations for facial expressions of emotion. Proceedings of the National Academy of Sciences of the United States of America, 99, 9072-9076. doi:10.1073/pnas.142165999

Pollak, S. D., Klorman, R., Thatcher, J. E., \& Cicchetti, D. (2001). P3b reflects maltreated children's reactions to facial displays of emotion. Psychophysiology, 38, 267274. doi:10.1111/1469-8986.3820267

Prinzie, P., Stams, G. J., Dekovic, M., Reigntjes, A. H., \& Belsky, J. (2009). The relations between parents' Big Five personality factors and parenting: A meta-analytic review. Journal of Personality and Social Psychology, 97, 351-362. doi:10.1037/a0015823

Roder, B. J., Bushnell, E. W., \& Sasseville, A. M. (2000). Infants' preferences for familiarity and novelty during the course of visual processing. Infancy, 1, 491-507. doi:10.1207/S15327078IN0104_9

Rothbart, M. K. (1981). Measurement of temperament in infancy. Child Development, 52, 569-578. doi:10.2307/ 1129176

Scott, L. S., \& Monesson, A. (2010). Experience-dependent neural specialization during infancy. Neuropsychologia, 48, 1857-1861. doi:10.1016/j.neuropsychologia.2010.02.008

Scott, L. S., \& Nelson, C. A. (2006). Featural and configural face processing in adults and infants: A behavioral and electrophysiological investigation. Perception, 35, 1107-1128. doi:10.1068/p5493

Shackman, J. E., Shackman, A. J., \& Pollak, S. D. (2007). Physical abuse amplifies attention to threat and increases anxiety in children. Emotion, 7, 838-852. doi:10.1037/1528-3542.7.4.838

Sinzig, J., Morsch, D., \& Lehmkuhl, G. (2008). Do hyperactivity, impulsivity and inattention have an impact on the ability of facial affect recognition in children with autism and ADHD? European Child $\mathcal{E}$ Adolescent Psychiatry, 17, 63-72. doi:10.1007/s00787-007-0637-9

Sorce, J. F., Emde, R. N., Campos, J. J., \& Klinnert, M. D. (1985). Maternal emotional signaling: Its effect on the visual cliff behavior of 1-year-olds. Developmental Psychology, 21, 195-200. doi:10.1037/0012-1649.21.1.195

Tottenham, N., Hare, T. A., Quinn, B. T., McCarry, T. W., Nurse, M., Gilhooly, T., . . . Casey, B. J. (2010). Prolonged institutional rearing is associated with atypically large amygdala volume and difficulties in emotion regulation. Developmental Science, 13, 46-61. doi:10.1111/ j.1467-7687.2009.00852.x

Walden, T. A., \& Ogan, T. A. (1988). The development of social referencing. Child Development, 59, 1230-1240. doi:10.2307/1130486

White, L. O., Wu, J., Borelli, J. L., Rutherford, H. J., David, D. H., Kim-Cohen, J., \& Crowley, M. J. (2012). Attachment dismissal predicts frontal slow-wave ERPs during rejection by unfamiliar peers. Emotion, 12, 690700. doi:10.1037/a0026750

Ziv, Y., Aviezer, O., Gini, M., Sagi, A., \& Koren-Karie, N. (2000). Emotional availability in the mother-infant dyad as related to the quality of infant-mother attachment relationship. Attachment and Human Development, 2, 149-169. doi:10.1080/14616730050085536

Ziv, Y., Gini, M., Guttman, S., \& Sagi, A. (1997). Dyadic emotional availability and quality of infant-mother attachment: A three point longitudinal study. Paper presented at the biennial meeting of the Society for Research in Child Development, Washington, DC. 